\title{
Learning a Manifold as an Atlas*
}

\author{
Nikolaos Pitelis \\ Chris Russell \\ School of EECS, Queen Mary, University of London \\ Lourdes Agapito \\ [nikolaos.pitelis, chrisr, lourdes] deecs.qmul.ac.uk
}

\begin{abstract}
In this work, we return to the underlying mathematical definition of a manifold and directly characterise learning a manifold as finding an atlas, or a set of overlapping charts, that accurately describe local structure. We formulate the problem of learning the manifold as an optimisation that simultaneously refines the continuous parameters defining the charts, and the discrete assignment of points to charts.

In contrast to existing methods, this direct formulation of a manifold does not require "unwrapping" the manifold into a lower dimensional space and allows us to learn closed manifolds of interest to vision, such as those corresponding to gait cycles or camera pose. We report state-ofthe-art results for manifold based nearest neighbour classification on vision datasets, and show how the same techniques can be applied to the $3 D$ reconstruction of human motion from a single image.
\end{abstract}

\section{Introduction}

One of the greatest challenges in computer vision lies in learning from high-dimensional data. In high-dimensional spaces, our day-to-day intuitions about distances are violated, and nearest neighbour (NN) and RBF based classifiers are less predictive and more easily swamped by noise. Manifold learning techniques attempt to avoid these difficulties by learning low dimensional representations of the data which preserve relevant information.

The majority of manifold learning techniques can be characterised as variants of kernel-PCA [9] that learn a smooth mapping from a high dimensional embedding space $\mathbb{R}^{n}$ into a low-dimensional space $\mathbb{R}^{d}$ while preserving properties of a local neighbourhood graph. ISOMAP [20] preserves global path distances; Locally Linear Embedding (LLE) [18] preserves the linear approximation of points by their nearest neighbours; Laplacian Eigenmaps [1] and Hessian LLE [5] preserve local Laplacian and Hessian derivatives of this graph respectively; while Local Tangent Space

* This research was funded by the European Research Council under the ERC Starting Grant agreement 204871-HUMANIS
Alignment (LTSA) [26] and Weighted LTSA (wLTSA) [25] preserve directions of greatest variance in each neighbourhood.

These methods rely on unwrapping the manifold into a single chart isomorphic to $\mathbb{R}^{d}$ to characterise it. As such, they have difficulty learning closed manifolds such as the surface of a ball or a Klein bottle. This is a fundamental limitation in computer vision where many of the manifolds of interest are closed or contain closed components. Views of an object under $360^{\circ}$ rotation of camera or lighting form a closed manifold; while the space of $3 \times 3$ image patches form a closed manifold similar to that of a Klein bottle [12], and in action recognition or 3D reconstruction, any repetitive action such as walking or running forms closed cycles.

In practice, existing manifold learning techniques can be adapted to handle closed cycles. For example, a one dimensional manifold of a gait cycle can be embedded in a two-dimensional space, and a larger neighbourhood that captures local second-order rather than the usual first-order information can be used to prevent the manifold from collapsing. We evaluate these standard approaches for $\mathrm{NN}$ based $3 \mathrm{D}$ reconstruction of walking and running, and find that they perform worse than using the original space.

The presence of noise causes further difficulties, as points now lie near, but not on the manifold we wish to recover. The local neighbourhood graph produced by $k$ nearest neighbours $(k-\mathrm{NN})$ is extremely vulnerable to the presence of noise, and the properties of the neighbourhood graph which are preserved by the embedding do not correspond to the underlying manifold. Here, many existing manifold learning methods fail severely and collapse into degenerate structures in the presence of noise (see Fig. 2).

In response to these difficulties, we present a novel formulation for manifold learning, which we refer to as learning an atlas. As is common in differential geometry, we characterise the manifold as an atlas, or set of overlapping charts, each of which is isomorphic to $\mathbb{R}^{d}$. Unlike existing machine learning approaches to manifold learning, this allows us to directly learn closed and partially closed manifolds. This differs from the standard formulations of differential geometry, which presuppose direct access to the 


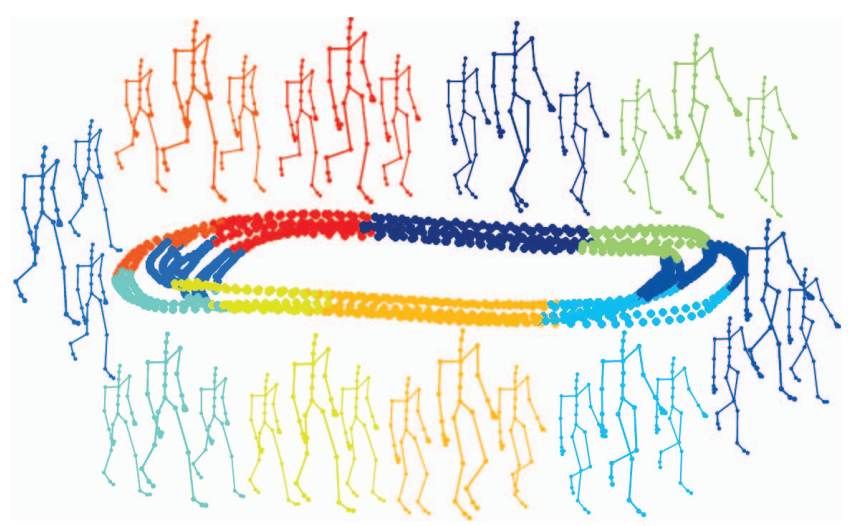

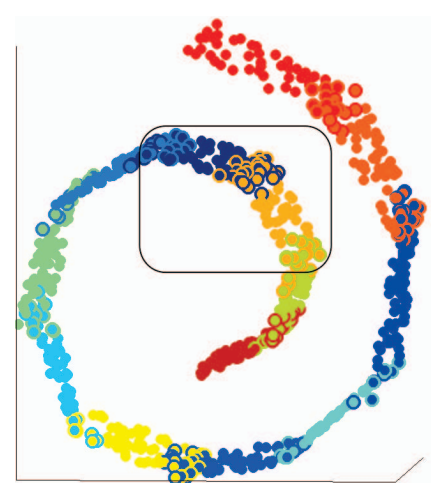

(a)

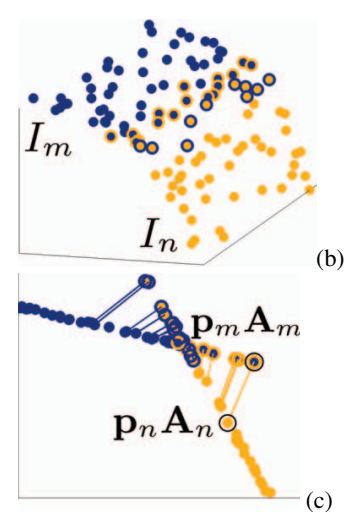

(b)

(c)

Figure 1. The overlapping charts found using Atlas. Left: The manifold of a gait cycle in the embedding space. Each colour indicates a different chart. Large stick-men represent the mean shape, while the small are \pm one standard deviation along the principal component. Right: A manifold as overlapping charts. (a) shows a typical decomposition into overlapping charts. A point's assignment to the interior of a chart is indicated by its colour. Membership of additional charts is indicated by a ring drawn around the point. (b) shows a detail of two overlapping charts. (c) shows a side view of the projected locations of the same point by different charts.

manifold; only in that we adaptively select the size of the region assigned to a chart in response to the amount of local noise, the intrinsic curvature of the manifold, and the sparsity of data. We formulate this problem as a hybrid continuous/discrete method that simultaneously optimises continuous parameters governing local charts, and the discrete assignment of points to those charts. We show how good solutions can be efficiently found using a combination of existing graph-cut based optimisation and PCA. Unlike methods that preserve unstable numerical properties of the local neighbourhood graph, our method only preserves coarse topological properties. Namely:

1. Any solution found using our approach has the property that if $p$ is a point lying on or near the manifold there exists a local chart $c_{i} \cong \mathbb{R}^{d}$ to which both $p$ and all of its neighbours are assigned ${ }^{1}$, and this allows the use of fast NN look up within $c_{i}$ for classification.

2. If two points $p$ and $q$ are path connected in the neighbourhood graph then any local charts $c_{p}$ and $c_{q}$ containing them will be path connected, in a dual graph of charts, in which charts are directly connected if and only if they share an assigned point in common. We make use of this property in manifold unwrapping.

As the method we propose finds charts corresponding to affine subspaces of the original space $\mathbb{R}^{n}$ we can directly use these for classification or for out-of-sample reconstruction from incomplete information. We illustrate this by demonstrating state-of-the-art results in NN based classification (Sec. 4), and 3D reconstruction from 2D tracks (Sec. 5).

The notion of a manifold as a collection of overlapping charts has been exploited in the literature. Both $[3,15]$ for-

\footnotetext{
${ }^{1}$ This is possible as the charts are overlapping and a point may belong to more than one chart.
}

mulated manifold learning as finding a set of charts that correspond to affine subspaces. As a final stage, both methods tried to align the subspaces found in $\mathbb{R}^{d}$, and cannot learn closed manifolds. In motion tracking, [14] performed agglomerative clustering over predefined affine subspaces to learn closed manifolds. Their approach made use of temporal information and was only applied to tracking.

In optimisation, the closest works to ours are the graphcut based [10, 16, 24] which adaptively grow and eliminate models to best fit the data. Within manifold learning, this adaptive shaping of regions is closest to methods such as [3], or the contraction and expansion (C\&E) method of [25] and the sparsity inducing method of [6], that adjust the neighbourhood before unwrapping. We extensively compare to the most recent of these $[6,25]$ in Sec. 4.

Outside of manifold learning, there is a strong relationship between our approach and methods of subspace clustering [22]. Like us, K-subspaces [21] alternates between assigning points to subspaces, and refitting the subspaces. While [21] keeps a fixed set of k subspaces, we start with an excess and discard those that do not explain the data.

\section{Formulation and optimisation}

We define an atlas $\mathcal{A}$, as a set of charts $\mathcal{A}=$ $\left\{c_{1}, c_{2}, \ldots c_{n}\right\}$, over points $\mathcal{P}$, with each chart $c_{i}$ containing a subset of points $P_{i} \subseteq \mathcal{P}$. Unlike the formal definition of a manifold that allows a chart to be warped as it is mapped into the embedding space using any continuous invertible mapping, we restrict ourselves to affine transforms. This restriction to affine transforms in approximating the local manifold does not limit the expressiveness of our approach ${ }^{2}$ and means that PCA can be used to find embeddings.

Our aim is to find a compact atlas containing few charts, such that: (i) for every point $p$, at least one chart contains

\footnotetext{
${ }^{2}$ Lie algebras make use of this equivalence between the two forms.
} 
both $p$ and a predefined neighbourhood ${ }^{3} \mathcal{N}_{p}$ of points in its vicinity, and (ii) the reconstruction error associated with mapping a point from its location in the chart back into the embedding space is as low as possible.

We decompose the problem of finding the best choice of atlas into two subproblems: Assigning points to charts subject to constraint $(i)$, and choosing the affine mappings to minimise reconstruction error. Given an initial excess of chart proposals (generated using PCA on random subsets of the data) we assign points to charts in such a way that they share points (Sec. 2.1), and alternate between refitting the subspaces associated with a chart (Sec. 2.2) and point reassignment.

\subsection{Point assignment to overlapping charts}

Similar assignment problems have been addressed in the context of model fitting by the Non-rigid Structure from Motion community [7, 16, 17], and we follow [16] in formulating this as an assignment of a set of models (or in our case charts) to each point, that minimises the accumulated reprojection error (summed over all points and all assigned models), and that satisfies constraint $(i)$. We define $I_{c}$, the interior of chart $c$, as the set of all points whose neighbours also belong to chart $c$; and we note that constraint $(i)$ is satisfied if and only if every point lies in the interior of some chart. We use $\mathbf{x}=\left\{\mathbf{x}_{1}, \ldots, \mathbf{x}_{P}\right\}$, where $\mathbf{x}_{p}$ refers to the set of charts assigned to point $p$, and seek an assignment that minimises:

$$
\underset{\mathbf{x} \in\left(2^{\mathcal{A}}\right)^{\mathcal{P}}}{\operatorname{argmin}} C(\mathbf{x})=\sum_{p \in \mathcal{P}}\left(\sum_{c \in \mathbf{x}_{p}} U_{p}(c)\right)+\lambda \operatorname{MDL}(\mathbf{x}),
$$

such that each point belongs to a chart's interior:

$$
\begin{array}{r}
\forall p \in \mathcal{P}, \exists c \in \mathcal{A}: p \in I_{c}, \\
p \in I_{c} \Longrightarrow \forall q \in \mathcal{N}_{p} c \in \mathbf{x}_{q},
\end{array}
$$

where $U_{p}(c)$ is the reconstruction error of assigning point $p$ to chart $c$, and

$$
\operatorname{MDL}(\mathbf{x})=\sum_{c \in \mathcal{A}} \Delta\left(\exists p \in I_{c}\right),
$$

where $\Delta(\cdot)$ denotes the Dirac indicator function that takes value 1 if $(\cdot)$ is true, and 0 otherwise. $\operatorname{MDL}(\mathbf{x})$ is a minimum description length prior [11] that penalises the total number of charts used in an assignment.

Despite the large state-space considered $\left(\left(2^{\mathcal{A}}\right)^{\mathcal{P}}\right.$ rather than the standard $\left.\mathcal{A}^{\mathcal{P}}\right)$, this can be solved with a variant of $\alpha$-expansion that assigns points to the interior of only one chart [16].

\footnotetext{
${ }^{3}$ In practice we take $\mathcal{N}_{p}$ to be the k-nearest neighbours of $p$
}

Pairwise regularisation In classification problems and when faced with relatively dense data, it can be useful to add additional regularisation terms to encourage larger charts that cover more of the manifold. Where necessary, we use the additional pairwise regularisation proposed in [17]. Writing $y_{p}$ for the assignment of point $p$ to the interior of one chart the regularisation takes the form of the standard Potts potential:

$$
\psi_{p, q}\left(y_{p}, y_{q}\right)=\theta \Delta\left(y_{p} \neq y_{q}\right),
$$

defined with constant weight $\theta>0$ over all edges in the $k$-NN graph. We use Atlas to refer to the method using the cost function of (1), and Atlas+ to refer to the same method with the additional pairwise regularisation of (5).

\subsection{Subspace costs and fitting}

We use $\mathbf{p}$ to refer to the vector location of point $p$. We define the $d$ dimensional subspace associated with chart $c_{i}$ in terms of its mean $\mu_{i}$, and an orthonormal matrix $\mathbf{C}_{i}$ which describes its principal directions of variance. We set the reconstruction error for point $p$ belonging to chart $c_{i}$ to

$$
U_{p}\left(c_{i}\right)=\left\|\mathbf{p}-\mu_{i}-\mathbf{C}_{i}^{T} \mathbf{C}_{i}\left(\mathbf{p}-\mu_{i}\right)\right\|_{2}^{2},
$$

i.e. the squared distance between a point and the backprojection of the closest vector on the chart $c_{i}$. Given a set of points $P_{i}$ assigned to chart $c_{i}$, the optimal parameters for minimising the reconstruction error $\sum_{p \in P_{i}} U_{p}\left(c_{i}\right)$ can be computed by setting $\mu_{i}$ to the mean of $P_{i}$, and $\mathbf{C}_{i}$ as the top $d$ eigenvectors of the covariance matrix of $P_{i}$.

In practice, both the subspaces corresponding to charts and the assignment of points to charts are initially unknown, so we bootstrap our solution by estimating an excess of possible subspaces, initialised by performing PCA on random samples as described above. Then, we perform a hill climbing approach that alternates between assigning points to charts, and refitting the subspaces to minimise the reconstruction error.

\section{Manifold unwrapping for visualisation}

Unlike existing approaches to manifold learning, our method has no requirement to unwrap the manifold and after characterising it as an atlas, as in the previous section, we can immediately perform classification (Sec. 4), or 3D reconstruction (Sec. 5). However, one of the principal uses of manifold learning $[18,20,26]$ is in creating a mapping from a high dimensional space into $\mathbb{R}^{2}$ or $\mathbb{R}^{3}$ suitable for visualising data. As a way of illustrating our method's robustness to sparse data, the presence of noise, and to systematic holes, we illustrate unwrapping on standard datasets.

Given a partitioning of the data into overlapping charts isomorphic to $\mathbb{R}^{d}$, for each affine chart $c_{i}$ we wish to find an affine mapping $A_{i}$ from $\mathbb{R}^{d} \rightarrow \mathbb{R}^{n}$ into the original space in 


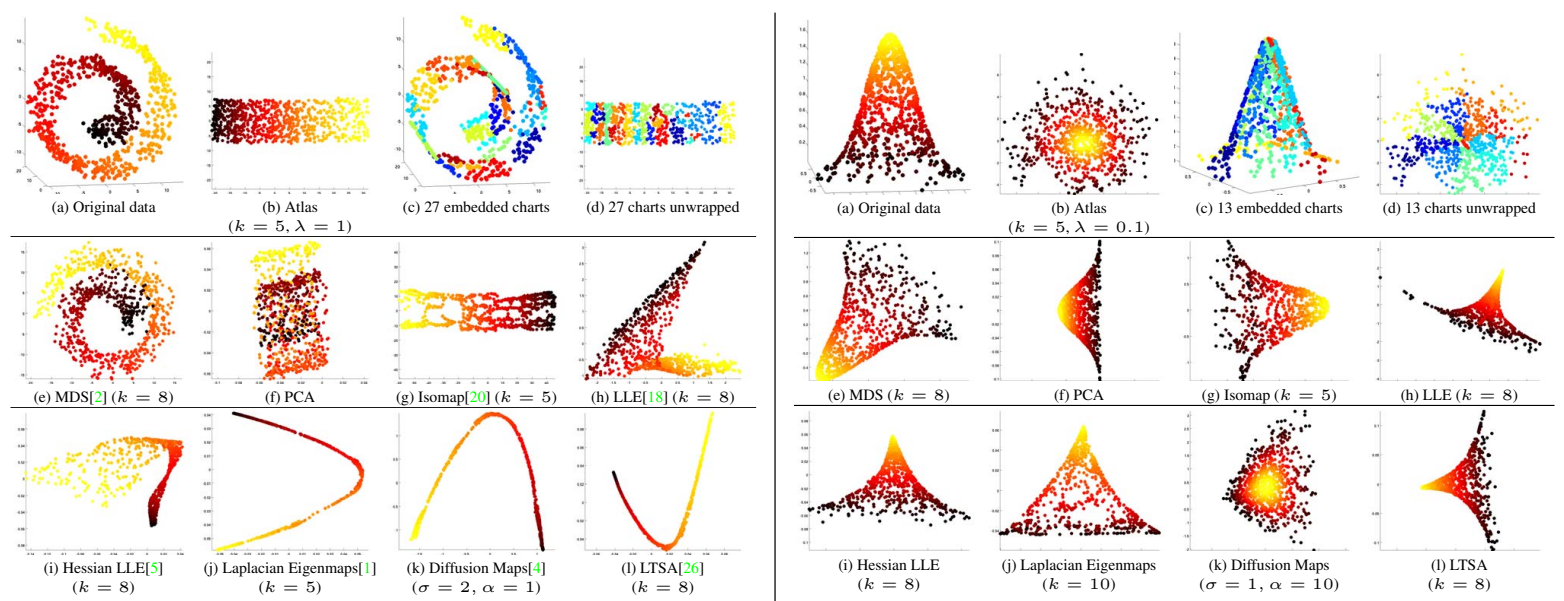

Figure 2. Unwrapping a Manifold Top row: (a) The original data; (b) the unwrapping generated by Atlas; (c) the charts found by Atlas; (d) the charts unwrapped. Other rows: (e-l) Other methods. All methods shown with optimal parameters. Left: Swiss Roll with Gaussian noise. Right: Sharply peaked Gaussian manifold with no noise. Additional examples in supplementary materials. Discussion in Sec. 3.1.

such a way that a point is projected into the same location by each subspace that it belongs to; the centres of each subspace are far from one another; the affine projection is not degenerate and distinct points in the subspace do not collapse on top of one another; and the reconstruction is as flat as possible, with most of the coefficients that do not correspond to a projection into the plane being small.

As neither visualisation nor unwrapping is a primary focus of the paper, we defer the precise form of the objective used to the supplementary materials and focus on results.

\subsection{Comparison with existing methods}

Fig. 2 illustrates the failure of existing methods, with optimally tuned parameters, on standard synthetic datasets ${ }^{4}$. Although the examples are straightforward for a human to unwrap -consisting of distinct 2D manifolds, with no self intersections, and lying in a 3D space- all methods except ours fail. For the Swiss roll this can be attributed to the Gaussian noise. As argued, if the points sampled lie not on the manifold, but near it, many of the properties of the $k$ $\mathrm{NN}$ graph (such as, in LLE, how points can be expressed as a weighted average of their neighbours) are unrepresentative of the underlying manifold and preserving these properties does not help in finding a good embedding. Our minimal assumption that neighbouring points in the $k$-NN graph should belong to the same subspace is robust to the presence of noise.

The failure of the Gaussian reconstruction is more representative of the general problems faced by any unwrapping method. Here, samples are more sparsely sampled in the tail of the Gaussian than in the central peak, and choosing a graph that is sufficiently connected in the tail leads to an over-connected graph about the peak, where the local $k$ $\mathrm{NN}$ graph characterises the local 3-dimensional structure instead of the 2D manifold. While such local degeneracies are

\footnotetext{
${ }^{4}$ Generated using MANI [23].
}

to be expected occasionally, for all nearest neighbours graph based methods, the local degeneracy spreads throughout the manifold and leads to a mapping in which the manifold is folded over itself. In the classification and reconstruction problems we discuss next, our method is used without unwrapping and such failures can never propagate throughout the manifold, and local mistakes remain local.

\section{Nearest neighbour classification}

Manifold learning classification is typically done by unwrapping the manifold and performing $1-\mathrm{NN}$ on the result. In our approach, we simply perform nearest-neighbour in each chart independently. During the manifold learning, each point $p$ is assigned by $\alpha$-expansion to the interior of a single chart, and we assign a label to $p$ simply by performing nearest neighbour in this chart. It is an empirical question whether we should include all points assigned to the chart when performing nearest neighbour or just those that belong to the interior of the chart. On the Yale Faces dataset, we observed that using only the interior points led to a small $(\sim 0.03 \%)$ consistent improvement, and we report all scores for Atlas using only interior points.

We compare against standard approaches LLE [18], LSTA [26], and PCA, and the recent state-of-the-art approaches of $[6,25]$. We evaluate our approach on face recognition on the Extended Yale Face Database B [8, 13], and the classification of handwritten digits on Semeion. In both cases, following [25], we randomly split the data for 100 folds and report average error.

SMCE [6] is a sparsity based method controlled by a parameter $\lambda$. The solution found provides local neighbourhood sizes, and dimensionality $d$. We report the score associated with the best value of $\lambda \in[0,100]$ that gives a manifold of dimensionality $d$. As SMCE [6] is intended to be used as a clustering algorithm, we report both clustering accuracy (SMCE clust), and 1-NN classification (SMCE NN). 

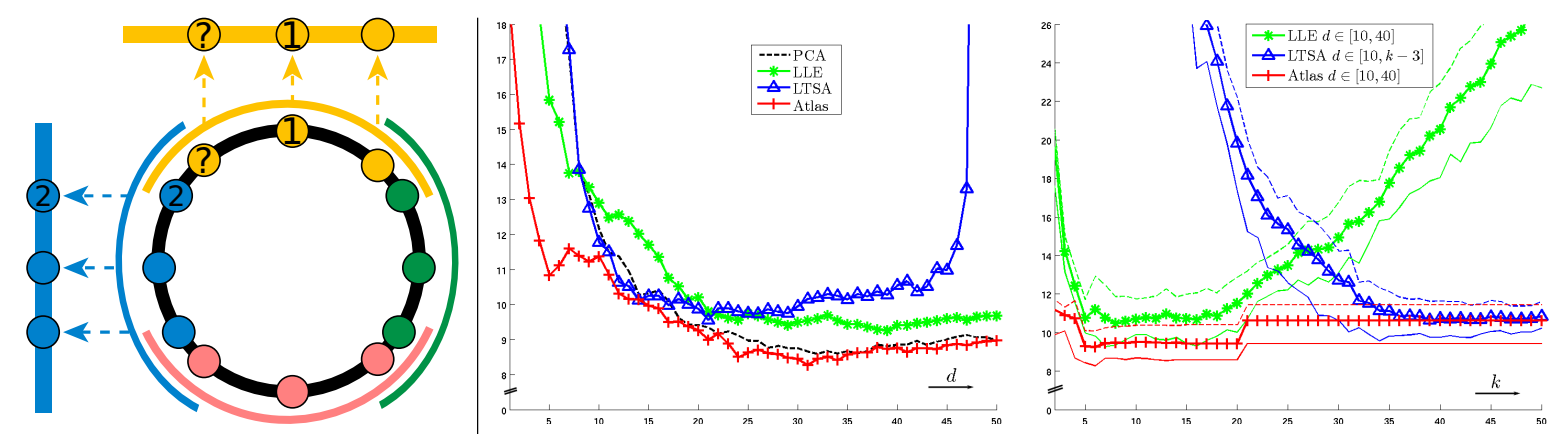

Figure 3. Left: Atlas based nearest neighbour. Points lie on the manifold (black circle). The manifold is decomposed into a set of charts (coloured straight lines) each of which approximates a local region of the embedding space (coloured curve) as an affine subspace. Interior points are mapped into the charts using the associated projection, and nearest neighbour look up is performed within each chart. Here you can see the point marked '?' has a nearest labelled neighbour of ' 1 ' in the chart space, rather than '2' in the original space. Centre: Minimal NN classification error(\%) on Semeion using the best value of $k$ for each choice of dimensionality. Right: Minimum(Fine), mean(Bold), and mean+standard deviation of NN classification error(\%) on Semeion, as $k$ varies. For Atlas the error is also averaged over $\lambda=10^{-1}, 10^{0}, \ldots, 10^{3}$.

Summary Across both datasets we substantially outperform wLTSA, LTSA, LLE, and SMCE. This is most noticeable in the Yale Faces dataset where our method Atlas+ obtains $0.70 \%$ error versus $3.01 \%$, the best reported error of [25], which used weighted LTSA with an adaptive neighbourhood, and $1.12 \%$ obtained by SMCE. The stability of our approach is also a substantial advantage; we obtain a classification accuracy of $0.71 \pm 0.01 \%$ for dimensionality 5-10 despite fixing to constant values the parameters $k, \lambda$, and $\theta$. On the Semeion dataset, we present a $1 \%$ improvement over wLTSA, LLE, and SMCE, again without varying $k$ or $\lambda$. In contrast, all other methods are tuned fully for each choice of dimensionality.

Extended Yale Face Database B contains 2,432 frontal face images of 38 individuals under a fixed pose and 64 illumination conditions. We pre-processed the original $192 \times 168$ images in the same way as [25]. Each image is resized to $32 \times 28$ and every pixel is represented as an 8 -bit binary vector. Moving around the 8-neighbourhood of each pixel, vector bits are set to 1 if its neighbouring pixels are of lower intensity and 0 otherwise. The vectors are concatenated to give a 7168-dimensional image vector. In each of the 100 folds, 16 images of each individual lie in the training set and 48 in the testing set.

Examination of the data provided by [25] showed a consistent "off by one" error in the image annotation. Images were provided ordered sequentially by individual, but rather than the expected 62 images, the first person had only 61 images associated with them, and every 1st image was consistently misclassified as belonging to the previous person. Visual inspection confirmed the "off by one" error, which has an approximate $1 \%$ impact on the results of [25]. Tables for uncorrected annotations are in the supplementary material, where we still consistently outperform other methods.

Table 1(a) shows 1-NN based face recognition in the original space and in lower-dimensional spaces of dimen- sionality 8 through 10 learnt with SMCE [6] while varying $\lambda$, and PCA, Atlas, and Atlas+ keeping their parameters fixed. Also shown are the uncorrected results of LLE, LTSA, and wLTSA given in [25], which provided state-ofthe-art results using Contraction and Expansion(C\&E) [25].

Table 1(b) shows the error of a 1-NN classifier using corrected image annotations, over [2-7]-dimensional spaces learnt with PCA, LLE, LTSA, SMCE, Atlas, and Atlas+, all using the same random splits. The neighbourhood size is tuned in $[2,20]$ for LLE and LTSA, while the parameters for Atlas and Atlas+ are fixed. Atlas outperforms PCA, LLE, LTSA, and SMCE clust for this range of $d$, with Atlas+ lowering the error further and outperforming all methods on all but one choices of $d$. Fig. 4 shows a representative sample of misclassified and correctly classified images. The majority of the images ( 94\%) are always classified correctly, while a small number of completely black images or heavily shadowed ones from specific subjects are consistently misclassified.

Semeion contains $159316 \times 16$ grey scale images of handwritten digits ( 0 to 9 ) written by approximately 80 people $^{5}$. We follow [25] in extracting a 256-dimensional binary vector from each image by applying a fixed threshold. In each split of the data 796 images are assigned to the training set and 797 to the testing set.

Table 2(a) contains the classification results we produced using PCA, SMCE, and Atlas, alongside results for LLE, LTSA, and wLTSA as reported in [25]. As neither the original folds, nor the code for wLTSA is available, we ran versions of LLE and LTSA provided by [23] on our folds, and confirmed that the performance was approximately the same. See supplementary materials for numbers.

As the performance of LLE and LTSA depends strongly on the choice of $k$ (see Fig. 3 centre), we select the best

\footnotetext{
5 available for download from http://archive.ics.uci.edu/ml/
} 


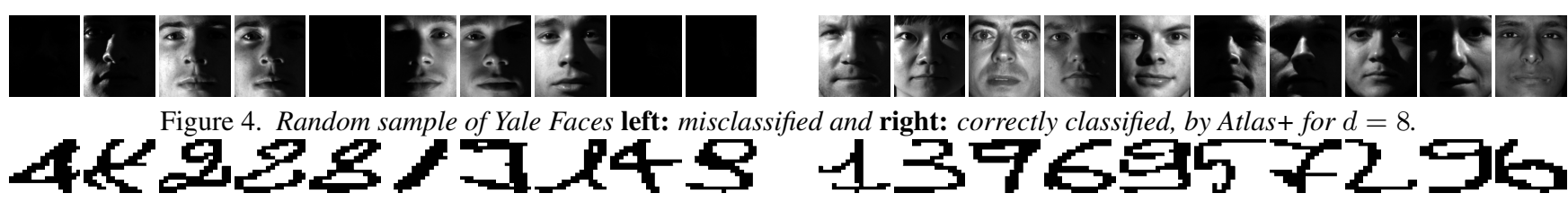

Figure 5. Random sample of Semeion images left: misclassified and right: correctly classified, by Atlas for $d=31$.

Table 1. Yale Faces classification error (\%) using 1-NN. The classification error of 1-NN in the original space is 4.39, and the lowest classification error of PCA $1-N N$ is 4.02 for $d=292$. \#c is the number of charts found by Atlas $(+)$. For LTSA and LLE, $k \in[2,20]$. SMCE uses $\lambda \in[1,100]$. For Atlas, $\lambda=700$ and $k=4$. For Atlas $+, \lambda=700, k=2$, and the pairwise terms use 5 neighbours with $\theta=1000$.

(a) $\operatorname{LLE}(\mathrm{C} \& \mathrm{E})$, $\operatorname{LTSA}(\mathrm{C} \& \mathrm{E})$, and $w \operatorname{LTSA}(\mathrm{C} \& \mathrm{E})$ as reported by $[25]$ versus PCA, SMCE, Atlas, and Atlas+.

\begin{tabular}{|c|c|c|c|c|c|c|c|c|}
\hline & PCA & $\begin{array}{l}\text { LLE } \\
\text { C\&E }\end{array}$ & $\begin{array}{l}\text { LTSA } \\
\text { C\&E }\end{array}$ & $\begin{array}{c}\text { wLTSA } \\
\text { C\&E }\end{array}$ & $\begin{array}{c}\text { SMCE } \\
\text { clust }\end{array}$ & $\begin{array}{c}\text { SMCE } \\
\text { NN }\end{array}$ & Atlas & Atlas+ \\
\hline$d$ & $\epsilon(\%)$ & $\epsilon(\%)$ & $\epsilon(\%)$ & $\epsilon(\%)$ & $\epsilon(\%) \lambda$ & $\epsilon(\%) \lambda$ & $\epsilon(\%) \# \mathrm{c}$ & $\epsilon(\%) \# \mathrm{c}$ \\
\hline 8 & 47.13 & 5.93 & 3.88 & 3.01 & 25.047 & 3.807 & $1.92 \quad 52$ & $\begin{array}{ll}\mathbf{0 . 7 0} & 67\end{array}$ \\
\hline 9 & 45.92 & 6.85 & 4.40 & 3.22 & 33.926 & 3.606 & 1.9558 & $\overline{\mathbf{0 . 7 1}} 69$ \\
\hline 10 & 42.23 & 6.59 & 4.18 & 3.13 & $47.50 \quad 4$ & 3.424 & 1.9058 & 0.71 69 \\
\hline
\end{tabular}

(b) PCA, LLE, LTSA, SMCE, Atlas, and Atlas+ results from our experiments for manifold dimensionality 2 to 7 .

\begin{tabular}{|c|c|c|c|c|c|c|c|}
\hline & PCA & LLE & LTSA & $\begin{array}{c}\text { SMCE } \\
\text { clust }\end{array}$ & $\begin{array}{c}\text { SMCE } \\
\text { NN }\end{array}$ & Atlas & Atlas+ \\
\hline$d$ & \begin{tabular}{|l}
$\epsilon(\%)$ \\
\end{tabular} & $\epsilon(\%) k$ & $\epsilon(\%) \quad k$ & $\epsilon(\%) \lambda$ & $\epsilon(\%) \lambda$ & $\epsilon(\%) \# \mathrm{c}$ & $\epsilon(\%) \# \mathrm{c}$ \\
\hline 2 & 97.53 & 3.374 & 8.225 & 11.8144 & 1.1263 & $\begin{array}{lll}1.99 & 112\end{array}$ & $\begin{array}{lll}0.80 & 65\end{array}$ \\
\hline 3 & 97.40 & 3.174 & 10.516 & 10.9023 & 1.2834 & $1.97 \quad 118$ & 1.9267 \\
\hline 4 & 88.18 & 3.084 & 16.789 & 11.5621 & 3.0321 & $1.93 \quad 129$ & $\mathbf{0 . 7 9} 69$ \\
\hline 5 & 73.60 & 2.334 & 10.8310 & 11.3113 & $4.35 \quad 14$ & $1.92 \quad 142$ & 0.71 69 \\
\hline 6 & 62.22 & 2.514 & $\begin{array}{ll}5.92 \quad 10\end{array}$ & 12.3811 & 4.7311 & $2.16 \quad 152$ & $\overline{0.71} 70$ \\
\hline 7 & 53.63 & 2.474 & $7.02 \quad 13$ & 17.239 & 4.369 & $2.25 \quad 160$ & $\overline{\mathbf{0 . 7 2}} 72$ \\
\hline
\end{tabular}

Table 2. Semeion dataset classification error (\%) of 1-NN classifier with PCA, LLE, LTSA, Atlas, and wLTSA. The classification error of 1-NN classifier in the original space is 10.92 with 10.90 reported in [25]. \#c denotes the number of charts found by Atlas.

(a) Columns 3, 4, and 5 contain the results reported in [25]. The best value of (b) Semeion dataset classification error (\%) of NN classifier with PCA, LLE, $k \in\{10,15,20,25\}$ for LLE and $k \in\{35,40,45,50\}$ for LTSA and wLTSA; LTSA, and Atlas. The best value of $k \in[2,50]$ for LLE and LTSA. For Atlas, For Atlas, $\lambda=100$ and $k=6$.

\begin{tabular}{|c|c|c|c|c|c|c|c|}
\hline & $\overline{P \text { PCA }}$ & $\overline{\text { LLE }}$ & \begin{tabular}{c|} 
LTSA \\
\end{tabular} & 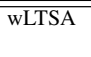 & $\begin{array}{c}\text { SMCE } \\
\text { clust }\end{array}$ & $\begin{array}{c}\text { SMCE } \\
\text { NN }\end{array}$ & Atlas \\
\hline$d$ & $\epsilon(\%)$ & $\epsilon(\%) \quad k$ & $\epsilon(\%) \quad k$ & $\epsilon(\%) \quad k$ & $\epsilon(\%) \quad \lambda$ & $\epsilon(\%) \quad \lambda$ & $\epsilon(\%) \# \mathrm{c}$ \\
\hline 12 & 11.37 & $12.47 \quad 10$ & 11.8945 & 11.0945 & $27.58 \quad 14$ & $\mathbf{9 . 2 6} 16$ & 10.854 \\
\hline 14 & 10.28 & $12.01 \quad 15$ & 10.2740 & $\mathbf{1 0 . 1 2} 40$ & $31.44 \quad 8$ & $10.77 \quad 8$ & 10.194 \\
\hline 16 & 10.41 & $11.67 \quad 10$ & 10.5350 & $\begin{array}{l}9.89 \quad 35\end{array}$ & 28.050 .89 & 11.720 .97 & $9.88 \quad 4$ \\
\hline 18 & 9.63 & 10.8510 & 10.0850 & $9.87 \quad 40$ & 28.610 .61 & $\begin{array}{lll}11.40 & 0.59\end{array}$ & $\begin{array}{cc}9.51 \quad 4 \\
\end{array}$ \\
\hline 20 & 9.41 & 10.2310 & $9.93 \quad 35$ & $9.85 \quad 50$ & 28.640 .27 & 11.590 .32 & 9.324 \\
\hline
\end{tabular}

$k \in[2,50]$ and report the lowest error for each choice of manifold dimensionality $d$. In contrast, for our method we fix our parameters to $k=6$ and $\lambda=100$ as we vary the local manifold dimensionality $d$. Nevertheless, Atlas outperforms all other methods for almost all choices of $d$. Table 2(b) shows additional results for $\operatorname{dim} 21-33$, where all methods except SMCE achieve their best performance.

The additional pairwise regularisation of Atlas+ does not help on this problem, with the best results for Atlas+ occurring as the pairwise regulariser $\theta \rightarrow 0$, at which point Atlas+ and Atlas are equivalent. Fig. 3 right shows a graph of results over a wide range of $k$. Atlas consistently outperforms other methods and is robust to a wide choice of dimensionality.

\section{Reconstructing human motion}

We apply our method to the task of non-rigid 3D reconstruction of human motion ${ }^{6}$ using 2D measurements acquired by a camera from a known viewpoint. Having learnt a manifold on 3D mocap data from one person using Atlas, we estimate the $3 \mathrm{D}$ pose of a new person directly from a test

\footnotetext{
${ }^{6} \mathrm{CMU}$ Motion Capture Database http://mocap.cs.cmu.edu/
} $\lambda=100$ and $k=6$.

\begin{tabular}{|c|c|c|c|c|c|c|c|c|}
\hline & PCA & LLE & LTSA & \multicolumn{2}{|c|}{$\begin{array}{c}\text { SMCE } \\
\text { clust }\end{array}$} & \multicolumn{2}{|c|}{$\begin{array}{c}\text { SMCE } \\
\text { NN }\end{array}$} & Atlas \\
\hline$d$ & $\epsilon(\%)$ & $\epsilon(\%) k$ & $\epsilon(\%) \quad k$ & $\epsilon(\%)$ & $\bar{\lambda}$ & $\epsilon(\%)$ & $\bar{\lambda}$ & $\epsilon(\%) \#$ \\
\hline 21 & 9.34 & $\begin{array}{ll}9.80 & 8\end{array}$ & $9.56 \quad 34$ & 29.17 & 0.22 & 11.67 & 0.2 & 8.99 \\
\hline 23 & 9.23 & 9.618 & $9.89 \quad 33$ & 35.55 & 0.05 & 12.02 & 0.05 & 8.90 \\
\hline 25 & 8.97 & 9.739 & $\begin{array}{ll}9.75 & 39\end{array}$ & - & - & - & - & 8.64 \\
\hline 27 & 8.77 & $9.57 \quad 8$ & $9.86 \quad 40$ & - & - & - & - & 8.61 \\
\hline 29 & 8.74 & $9.41 \quad 16$ & $9.75 \quad 42$ & - & - & - & - & 8.48 \\
\hline 31 & 8.66 & $9.54 \quad 8$ & 10.1643 & - & - & - & - & 8.27 \\
\hline 33 & 8.67 & 9.6916 & 10.2944 & - & - & - & - & 8.50 \\
\hline
\end{tabular}

image showing the 2D image locations of the mocap markers. This is an inverse problem of reconstructing the $3 \mathrm{D}$ pose given 2D input data and knowledge of our piecewise affine manifold.

Assuming an orthographic camera of known orientation, both the affine subspaces of the manifold and the backprojection of points into 3D form hyper-planes. The problem of 3D reconstruction from 2D image data can then be posed as out-of-sample reconstruction, or finding the point lying on any of the affine subspaces that is closest to the back-projected hyper-plane. This can be done by an exhaustive search over the subspaces ${ }^{7}$, and finding the closest points on the two hyper-planes, which can be done using standard linear algebra e.g. [19]. The closest point on the manifold subspaces is picked as the $3 \mathrm{D}$ reconstruction.

This technique cannot be directly applied to standard manifold learning methods, such as LLE and LTSA, which do not provide an explicit embedding of the manifold in the original space. For these methods, we learn the motion manifold using the 2D joint locations of all training and test sequences, then reconstruct by finding the nearest neigh-

\footnotetext{
${ }^{7}$ This is fast, as typically, less than 10 subspaces are used to characterise a gait cycle. See Fig. 1.
} 


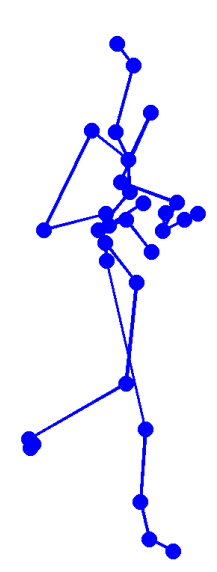

2D Input

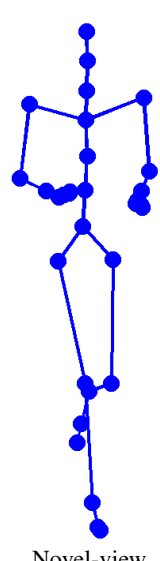

Ground Truth Gaussian noise added to both the image reconstructed and the training data. The reconstructions of $21-N N$ and Atlas have average $R M S$ reconstruction error $(\epsilon)$ of $5 \mathrm{~cm}$ and $3.74 \mathrm{~cm}$ respectively. Marker colours are based on squared reconstruction error.

bours in the low-dimensional space and averaging their 3D coordinates in the original space. In a similar way, we perform $k$-NN reconstruction in the 3D projection of the original space. Reconstruction using PCA is done by treating it as a degenerate case of our method with only one subspace.

For evaluation we selected two subsets of the CMU mocap database; dataset I containing walking sequences and dataset II containing both walking and running sequences. Dataset I uses as training set 4 walking sequences of one subject and as testing set 8 sequences of two different subjects. Dataset II has a training set of 4 walking sequences of one subject and 4 running sequences of another subject, testing contains 16 sequences of two different subjects, 4 walking and 4 running from each. The test images are generated by projecting the 31 mocap markers onto each frame using an orthographic camera model. The image measurements are registered to the centroid of each image to eliminate the translation component. Each walking or running sequence includes about 4 cycles of motion. The subjects used for training and testing do not overlap. Gaussian noise is added to the original 3D data used in training. We add 5 different levels of Gaussian noise with standard deviation $\sigma$ ranging from $0.5 \mathrm{~cm}$ to $5 \mathrm{~cm}$.

We show reconstruction results for PCA, $k$-NN, and Atlas. The error shown in all cases is the root mean squared $3 \mathrm{D}$ reconstruction error (RMS) in $\mathrm{cm}$, averaged over all markers and frames in the testing set. For our method, we keep the parameters fixed for each dimensionality across all levels of noise, with $\lambda \in\left\{10^{0}, 10^{1}, \ldots, 10^{6}\right\}$ and $k \in[2,21]$. For $k$-NN we show both the best $k$ per noise level and the value of $k$ that gives the lowest error averaged for all levels of noise.

Fig. 7 shows comparative results of the 3D reconstruction error for PCA, $k$-NN, and Atlas when the scene is

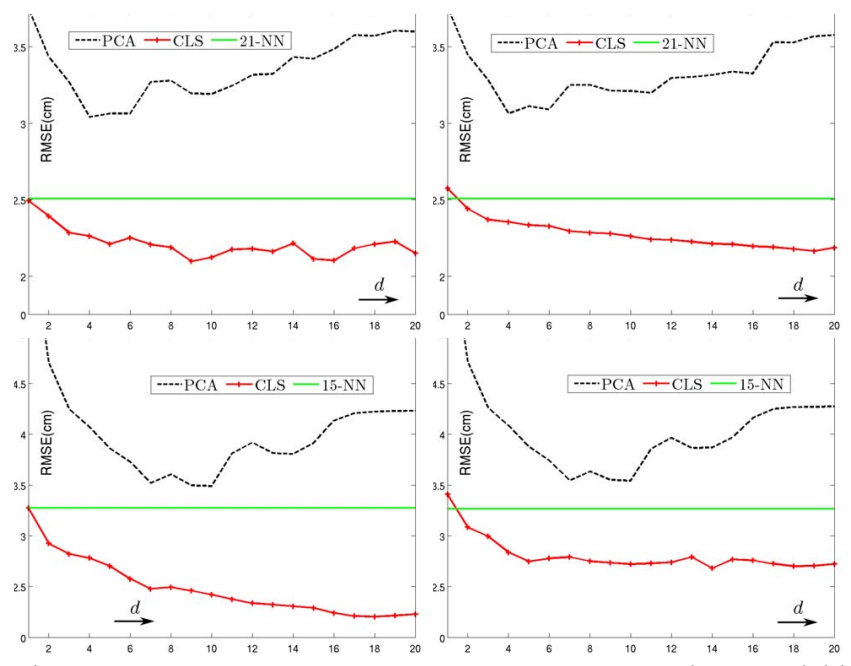

Figure 7. CMU average RMS reconstruction error for manifold dimensionality 1 to 20 . Top row: dataset I walking sequences and bottom row: dataset II walking and running sequences. Left column shows $0 \mathrm{~cm}$ noise case and right column $2 \mathrm{~cm}$ noise.

viewed from a generic view-point on datasets I (top) and II (bottom). We show results for varying values of the dimensionality $d$ in the noise-less case (left) and the case of noisy data with $\sigma=2 \mathrm{~cm}$ (right). Atlas clearly outperforms all other methods and, unlike PCA, its performance does not degrade as the dimensionality increases.

Fig. 6 shows the reconstruction results of a running sequence with $3 \mathrm{~cm}$ Gaussian noise. Table 3 shows more extensive comparisons: with 6 levels of noise and local dimensionality $d \in[1,20]$; and two different camera view-points: a side view and a generic view-point. The same pattern can be observed of our method outperforming all others.

We observed experimentally that $k$-NN on the manifold spaces learnt by LLE and LTSA had consistently poorer performance than $k$-NN in the original space and their results are omitted.

\section{Conclusion}

Starting from the formal definition of a manifold, as an atlas of overlapping charts, we have presented a novel approach to manifold learning that directly characterises the manifold in the original embedding space. In comparison with existing methods, our method has substantial theoretical advantages. In particular, unlike existing methods, it can potentially learn any form of manifold rather than being restricted to manifolds that can be expressed by a single chart. We show a substantial boost in performance classification and robustness to the choice of both manifold dimensionality and neighbourhood graph.

Unlike existing approaches, our characterisation of the manifold directly in the embedding space allows us to recover previously unseen and partially missing data easily, and we have shown applications in the reconstruction of hu- 
Table 3. CMU average RMS reconstruction error in $\mathrm{cm}$. Noise standard deviation in $\mathrm{cm}$.

\begin{tabular}{|c|c|c|c|c|c|c|}
\hline \multicolumn{7}{|c|}{ dataset I walking sequences } \\
\hline noise & 0 & 0.5 & , & 2 & 3.5 & 5 \\
\hline$d$ & PCA Atlas & PCA Atlas & PCA Atlas & PCA Atlas & PCA Atlas & PCA Atlas \\
\hline 1 & $\begin{array}{ll}.46 \quad \mathbf{2 . 3 9} \\
\end{array}$ & $3.46 \quad \mathbf{2 . 4 0}$ & $3.46 \quad \mathbf{2 . 4 3}$ & $3.47 \quad \mathbf{2 . 5 2}$ & $3.48 \quad \mathbf{2 . 7 7}$ & $3.51 \quad 2.99$ \\
\hline 5 & $2.51 \quad \mathbf{1 . 9 9}$ & $2.51 \quad \mathbf{2 . 0 0}$ & $2.52 \quad 2.09$ & $2.55 \quad 2.13$ & $2.64 \quad 2.41$ & $2.79 \quad \mathbf{2 . 6 4}$ \\
\hline 10 & $\begin{array}{ll}1.98 & \mathbf{1 . 8 7}\end{array}$ & $1.99 \quad \mathbf{1 . 8 7}$ & $2.00 \quad 1.88$ & $2.06 \quad \mathbf{2 . 0 3}$ & $2.29 \quad 2.33$ & 2.69 \\
\hline 15 & $\begin{array}{ll}1.85 & 1.85\end{array}$ & $1.86 \quad 1.86$ & $1.88 \quad \mathbf{1 . 8 6}$ & $1.96 \quad 1.96$ & $2.31 \quad 2.31$ & 2.75 \\
\hline 20 & & & & $1.90 \quad 1.90$ & $\mathbf{2 . 3 4} \quad 2.37$ & 2.88 \\
\hline $21-\mathrm{NN}$ & $\epsilon 2.47$ & $\epsilon 2.47$ & $\epsilon 2.47$ & $\epsilon 2.47$ & $\epsilon 2.56$ & $\epsilon 2.80$ \\
\hline$k$-NN & $\epsilon 2.43 k 7$ & $\epsilon 2.43 k 7$ & $\epsilon 2.44 k 8$ & $\epsilon 2.46 k 13$ & $\epsilon 2.56 \quad k 21$ & $\epsilon 2.80 k 21$ \\
\hline \multicolumn{7}{|c|}{ generic view-point } \\
\hline noise & 0 & 0.5 & & 2 & 3.5 & 5 \\
\hline$d$ & PCA Atlas & PCA Atlas & PCA Atlas & $\begin{array}{ll}\text { PCA } & \text { Atlas } \\
\end{array}$ & $\begin{array}{ll}\text { PCA } & \text { Atlas } \\
\end{array}$ & PCA Atlas \\
\hline 1 & $3.75 \quad \mathbf{2 . 4 9}$ & $\begin{array}{ll}3.75 & \mathbf{2 . 4 9}\end{array}$ & $3.75 \quad \mathbf{2 . 5 0}$ & $\begin{array}{ll}3.76 & \mathbf{2 . 5 7}\end{array}$ & $3.76 \quad \mathbf{2 . 8 2}$ & $3.79 \quad \mathbf{3 . 0 5}$ \\
\hline 5 & & & $3.07 \quad 2.25$ & $3.11 \quad 2.33$ & & 3.21 \\
\hline 10 & $3.19 \quad 2.12$ & $3.20 \quad 2.11$ & $3.18 \quad 2.19$ & $3.21 \quad 2.26$ & $3.35 \quad 2.50$ & 3.40 \\
\hline 15 & $3.42 \quad 2.11$ & $3.43 \quad 2.08$ & $3.43 \quad 2.13$ & $3.34 \quad 2.21$ & $3.55 \quad 2.49$ & 3.48 \\
\hline 20 & $3.60 \quad 2.15$ & $3.60 \quad \mathbf{2 . 0 4}$ & $3.64 \quad 2.10$ & $3.58 \quad 2.18$ & $3.61 \quad \mathbf{2 . 5 0}$ & $3.74 \quad \mathbf{3 . 0 6}$ \\
\hline $21-\mathrm{NN}$ & \multirow{2}{*}{$\begin{array}{c}\epsilon 2.51 \\
\epsilon 2.49 k 9\end{array}$} & $\epsilon 2.50$ & \multirow{2}{*}{$\begin{array}{c}\epsilon 2.50 \\
\epsilon 2.48 k 9\end{array}$} & $\epsilon 2.51$ & $\epsilon 2.62$ & $\epsilon 2.89$ \\
\hline$k$-NN & & $\epsilon 2.48 k 8$ & & $\epsilon 2.50 k 14$ & $\epsilon 2.62 \quad k 21$ & $\epsilon 2.89 \quad k 21$ \\
\hline \multicolumn{7}{|c|}{ dataset II walking and running sequences } \\
\hline \multicolumn{7}{|c|}{ side view } \\
\hline noise & 0 & 0.5 & 1 & 2 & 3.5 & 5 \\
\hline$d$ & PCA Atlas & PCA Atlas & PCA Atlas & PCA Atlas & $\begin{array}{ll}\text { PCA } & \text { Atlas } \\
\end{array}$ & PCA Atlas \\
\hline 1 & $\begin{array}{ll}5.85 & 3.14\end{array}$ & $\begin{array}{ll}5.85 & \mathbf{3 . 1 6} \\
\end{array}$ & $\begin{array}{ll}5.85 & \mathbf{3 . 1 4}\end{array}$ & $\begin{array}{ll}5.86 & \mathbf{3 . 3 3} \\
\end{array}$ & $\begin{array}{ll}5.87 & \mathbf{3 . 6 3} \\
\end{array}$ & $\begin{array}{ll}5.87 & \mathbf{3 . 7 5}\end{array}$ \\
\hline 5 & $3.28 \quad \mathbf{2 . 4 3}$ & $3.28 \quad \mathbf{2 . 4 3}$ & $3.29 \quad \mathbf{2 . 4 3}$ & $3.31 \quad \mathbf{2 . 5 2}$ & $3.38 \quad \mathbf{2 . 7 5}$ & 3.49 \\
\hline 10 & $2.60 \quad 2.30$ & $2.61 \quad 2.34$ & $2.62 \quad 2.34$ & $2.66 \quad \mathbf{2 . 4 2}$ & $2.86 \quad \mathbf{2 . 6 7}$ & $3.10 \quad \mathbf{2 . 9 6}$ \\
\hline 15 & $2.61 \quad 2.25$ & $2.61 \quad 2.26$ & $2.63 \quad 2.29$ & $2.69 \quad \mathbf{2 . 3 9}$ & $2.91 \quad 2.64$ & 3.26 \\
\hline 20 & $2.55 \quad \mathbf{2 . 4 4}$ & $2.56 \quad 2.24$ & $2.58 \quad 2.23$ & $2.66 \quad \mathbf{2 . 3 9}$ & $2.95 \quad \mathbf{2 . 6 8}$ & $3.39 \quad \mathbf{3 . 0 3}$ \\
\hline 13-NN & $\epsilon 3.17$ & $\epsilon 3.17$ & $\epsilon 3.17$ & $\epsilon 3.17$ & $\epsilon 3.29$ & $\epsilon 3.49$ \\
\hline$k$-NN & $\epsilon 3.12 k 5$ & $\epsilon 3.12 k 5$ & $\epsilon 3.12 k 5$ & $\epsilon 3.15 \mathrm{kf}$ & $\epsilon 3.28 \quad k 18$ & $\epsilon 3.40 \quad k 21$ \\
\hline & & & generic vi & -point & & \\
\hline noise & 0 & 0.5 & 1 & 2 & 3.5 & 5 \\
\hline$d$ & PCA Atlas & PCA Atlas & PCA Atlas & PCA Atlas & PCA Atlas & PCA Atlas \\
\hline 1 & $\begin{array}{ll}6.08 & \mathbf{3 . 2 7}\end{array}$ & \begin{tabular}{ll|}
6.08 & $\mathbf{3 . 3 1}$
\end{tabular} & $\begin{array}{ll}6.08 & \mathbf{3 . 3 4} \\
\end{array}$ & $\begin{array}{|ll|}6.09 & \mathbf{3 . 4 1} \\
\end{array}$ & $\begin{array}{ll}6.09 & \mathbf{3 . 7 0}\end{array}$ & $\begin{array}{ll}6.09 & \mathbf{3 . 8 2}\end{array}$ \\
\hline 5 & $\begin{array}{ll}3.86 & \mathbf{2 . 7 0}\end{array}$ & $3.86 \quad \mathbf{2 . 6 9}$ & $\begin{array}{ll}3.86 & \mathbf{2 . 7 0}\end{array}$ & $3.88 \quad \mathbf{2 . 7 5}$ & $3.94 \quad 3.01$ & 4.01 \\
\hline 10 & $3.49 \quad \mathbf{2 . 4 2}$ & $3.49 \quad \mathbf{2 . 5 2}$ & $3.50 \quad 2.62$ & $3.54 \quad 2.72$ & $3.68 \quad 3.20$ & 3.82 \\
\hline 15 & $3.91 \quad 2.29$ & $3.92 \quad \mathbf{2 . 4 1}$ & $3.97 \quad \mathbf{2 . 5 8}$ & $3.97 \quad 2.77$ & $4.04 \quad \mathbf{3 . 1 8}$ & $4.28 \quad \mathbf{3 . 5 4}$ \\
\hline 20 & $\begin{array}{ll}4.23 & \mathbf{2 . 2 3} \\
\end{array}$ & $\begin{array}{ll}4.23 \quad 2.37 \\
\end{array}$ & $\begin{array}{ll}4.24 & \mathbf{2 . 4 8} \\
\end{array}$ & $\begin{array}{ll}4.27 \quad 2.72 \\
\end{array}$ & $\begin{array}{ll}4.30 & \mathbf{3 . 1 8} \\
\end{array}$ & $\begin{array}{ll}4.43 \quad 3.67 \\
\end{array}$ \\
\hline $15-\mathrm{NN}$ & $\epsilon 3.27$ & $\epsilon 3.28$ & $\epsilon 3.27$ & $\epsilon 3.27$ & $\epsilon 3.38$ & $\epsilon 3.55$ \\
\hline$k$-NN & $\epsilon 3.24 k 4$ & $\epsilon 3.24 k 5$ & $\epsilon 3.24 k 6$ & $\epsilon 3.25 \mathrm{k} 8$ & $\epsilon 3.38 k 20$ & $\epsilon 3.49 k 21$ \\
\hline
\end{tabular}

man motion.

\section{References}

[1] M. Belkin and P. Niyogi. Laplacian eigenmaps and spectral techniques for embedding and clustering. Advances in neural information processing systems, 14:585-591, 2001. 1, 4

[2] I. Borg and P. Groenen. Modern multidimensional scaling: Theory and applications. Springer, 2005. 4

[3] M. Brand. Charting a manifold. In Advances in Neural Information Processing Systems, pages 961-968, 2003. 2

[4] R. R. Coifman and S. Lafon. Diffusion maps. Applied and Computational Harmonic Analysis, 21(1):5 - 30, 2006. 4

[5] D. Donoho and C. Grimes. Hessian eigenmaps: Locally linear embedding techniques for high-dimensional data. Proceedings of the National Academy of Sciences, 100(10):5591-5596, 2003. 1, 4

[6] E. Elhamifar and R. Vidal. Sparse manifold clustering and embedding. In Advances in Neural Information Processing Systems, pages 55-63, 2011. 2, 4, 5

[7] J. Fayad, C. Russell, and L. Agapito. Automated articulated structure and $3 \mathrm{~d}$ shape recovery from point correspondences. In International Conference in Computer Vision, 2011. 3

[8] A. Georghiades, P. Belhumeur, and D. Kriegman. From few to many: Illumination cone models for face recognition under variable lighting and pose. IEEE Transactions on Pattern Analysis and Machine Intelligence, 23(6):643-660, 2001. 4
[9] J. Ham, D. Lee, S. Mika, and B. Schölkopf. A kernel view of the dimensionality reduction of manifolds. In Proceedings of the twenty-first international conference on Machine learning, page 47. ACM, 2004. 1

[10] H. Isack and Y. Boykov. Energy-based geometric multimodel fitting. International Journal of Computer Vision (IJCV), 97(2), 2012. 2

[11] L. Ladicky, C. Russell, P. Kohli, and P. Torr. Graph cut based inference with co-occurrence statistics. In European Conference on Computer Vision, 2010. 3

[12] A. B. Lee, K. S. Pedersen, and D. Mumford. The nonlinear statistics of high-contrast patches in natural images. International Journal of Computer Vision, 54(1-3), 2003. 1

[13] K. Lee, J. Ho, and D. Kriegman. Acquiring linear subspaces for face recognition under variable lighting. IEEE Transactions on Pattern Analysis and Machine Intelligence, 27(5):684-698, 2005. 4

[14] J. C. Nascimento and J. G. Silva. Manifold learning for object tracking with multiple motion dynamics. In European Conference on Computer Vision, pages 172-185, 2010. 2

[15] S. Roweis, L. K. Saul, G. E. Hinton, et al. Global coordination of local linear models. Advances in neural information processing systems, 2:889-896, 2002. 2

[16] C. Russell, J. Fayad, and L. Agapito. Energy based multiple model fitting for non-rigid structure from motion. In Computer Vision and Pattern Recognition, 2011. 2, 3

[17] C. Russell, J. Fayad, and L. Agapito. Dense non-rigid structure from motion. 3dimPVT, 2012. 3

[18] L. Saul and S. Roweis. Think globally, fit locally: unsupervised learning of low dimensional manifolds. The Journal of Machine Learning Research, 4:119-155, 2003. 1, 3, 4

[19] G. Strang. Introduction to Linear Algebra. WellesleyCambridge Press, 1993. 6

[20] J. B. Tenenbaum, V. de Silva, and J. C. Langford. A global geometric framework for nonlinear dimensionality reduction. Science, 2000. 1, 3, 4

[21] P. Tseng. Nearest q-flat to $\mathrm{m}$ points. Journal of Optimization Theory and Applications, 105(1):249-252, April 2000. 2

[22] R. Vidal. Subspace clustering. IEEE Signal Processing Magazine, 28(2):52-68, 2011. 2

[23] T. Wittman. Manifold learning matlab demo, 2005. URL: http://www.math.umn.edu/ wittman/mani/index.html. 4, 5

[24] R. Zabih and V. Kolmogorov. Spatially coherent clustering using graph cuts. In Computer Vision and Pattern Recognition, pages 437-444, 2004. 2

[25] Z. Zhang, J. Wang, and H. Zha. Adaptive manifold learning. Pattern Analysis and Machine Intelligence, IEEE Transactions on, 34(2):253 -265, feb. 2012. 1, 2, 4, 5, 6

[26] Z. Zhang and H. Zha. Principal manifolds and nonlinear dimension reduction via local tangent space alignment. SIAM Journal of Scientific Computing, 26:313-338, 2002. 1, 3, 4 